\title{
On a Simple Relation between Duffin-Kemmer-Petiau and Tzou Algebras
}

\author{
Andrzej Okniński (iD \\ Chair of Mathematics and Physics, Politechnika Świętokrzyska, Al. 1000-lecia PP 7, 25-314 Kielce, Poland \\ Correspondence should be addressed to Andrzej Okniński; fizao@tu.kielce.pl
}

Received 3 April 2018; Accepted 28 June 2018; Published 22 July 2018

Academic Editor: Carlo Bianca

Copyright (C) 2018 Andrzej Okniński. This is an open access article distributed under the Creative Commons Attribution License, which permits unrestricted use, distribution, and reproduction in any medium, provided the original work is properly cited.

A simple link between $\beta^{\mu}$ matrices of the Duffin-Kemmer-Petiau theory and $\rho^{\mu}$ matrices of Tzou representations is constructed. The link consists of a constant unitary transformation of the $\beta^{\mu}$ matrices and a projection onto a lower-dimensional subspace.

\section{Introduction}

The Duffin-Kemmer-Petiau (DKP) equations [1-3] have been gaining importance due to their applications to problems in particle and nuclear physics of spin 0 and spin 1 mesons [415]. Since spin 1 bosons can be also described by HagenHurley equations [16-18] involving Tzou algebra [19-21], relations between DKP and Tzou algebras deserve a separate study.

Recently, we have constructed a similarity transformation that maps the free Duffin-Kemmer-Petiau equations to equations involving the Tzou algebras [22].

However, this similarity transformation is rather complicated, is momentum dependent, and transforms the whole operator $\left(\beta^{\mu} k_{\mu}-m\right)$ only. In the present paper a simple link between $\beta^{\mu}$ matrices of the DKP theory and $\rho^{\mu}$ matrices of Tzou representations is constructed. The link consists of a (constant) unitary transformation of the $\beta^{\mu}$ matrices and a projection onto a lower-dimensional subspace.

The inspiration has come from our earlier experience in splitting relativistic equations within the spinor formalism. It turns out that the process of splitting the DKP equations leads to subequations involving Tzou algebras; see [19] and references therein. Therefore, our first step to find a simple relation between $\beta^{\mu}$ and $\rho^{\mu}$ matrices consists in transition to spinor formalism via a unitary transformation.

In the next section, Tzou and DKP algebras are described in the setting of relativistic wave equations. In Sections 3 and 4 the $\beta^{\mu}$ matrices are converted by application of unitary transformations and projection operators into two sets of Tzou matrices $\rho^{\mu}$, one of them having been used in the Hagen-Hurley equations describing spin 1 bosons [16-19]. We discuss our results in the last section. In what follows we use notation and conventions described in [19].

\section{Tzou and Duffin-Kemmer-Petiau Algebras in Relativistic Wave Equations}

Relativistic equations describing elementary particles can be written as

$$
\rho^{\mu} p_{\mu} \Psi=m \Psi
$$

where $p_{\mu} \stackrel{d f}{=} i\left(\partial / \partial x^{\mu}\right)$ and $\rho^{\mu}$ are some matrices.

Equation (1) describes a particle with definite mass on condition that $\rho^{\mu}$ obey the Tzou commutation relations [20, 21, 23-25]:

$$
\sum_{\lambda, \mu, \nu}^{\lambda} \rho^{\lambda} \rho^{\mu} \rho^{\nu}=\sum_{\lambda, \mu, \nu} g^{\lambda \mu} \rho^{\nu},
$$

where we sum over all permutations of $\lambda, \mu, \nu$.

Important solution of the Tzou conditions (2) was constructed in [1] in form

$$
\rho^{\mu}=\frac{1}{2}\left(\gamma^{\mu} \otimes I_{4 \times 4}+I_{4 \times 4} \otimes \gamma^{\mu}\right) \equiv \beta^{\mu} .
$$


Such $\beta^{\mu}$ obey simpler but more restrictive commutation relations $[1,2]$ :

$$
\beta^{\lambda} \beta^{\mu} \beta^{\nu}+\beta^{\nu} \beta^{\mu} \beta^{\lambda}=g^{\lambda \mu} \beta^{\nu}+g^{\nu \mu} \beta^{\lambda},
$$

for which (1) leads to the Duffin-Kemmer-Petiau (DKP) theory of spin 0 and 1 mesons; see [1-3]. This 16-dimensional representation (3) of $\beta^{\mu}$ matrices (denoted as 16) is reducible and can be decomposed as $\mathbf{1 6}=\mathbf{1 0} \oplus \mathbf{5} \oplus \mathbf{1}$. Representation 10 (spin 1 case) is realized in terms of $10 \times 10$ matrices, while representation 5 (spin 0 ) involves $5 \times 5$ matrices; see $[1,23,24]$.

In the case of $5 \times 5(\operatorname{spin}-0)$ representation of $\beta^{\mu}$ matrices (1) is equivalent to the following set of equations:

$$
\begin{aligned}
p^{\mu} \psi & =m \psi^{\mu} \\
p_{\nu} \psi^{\nu} & =m \psi,
\end{aligned}
$$

if we define $\Psi$ in (1) as

$$
\Psi=\left(\psi^{\mu}, \psi\right)^{T}=\left(\psi^{0}, \psi^{1}, \psi^{2}, \psi^{3}, \psi\right)^{T},
$$

where ${ }^{T}$ denotes transposition of a matrix [19].

In the case of $10 \times 10$ (spin-1) representation of matrices $\beta^{\mu}(1)$ reduces to

$$
\begin{aligned}
p^{\mu} \psi^{\nu}-p^{\nu} \psi^{\mu} & =m \psi^{\mu \nu} \\
p_{\mu} \psi^{\mu \nu} & =m \psi^{\nu},
\end{aligned}
$$

with the following definition of $\Psi$ in (1):

$$
\begin{aligned}
\Psi & =\left(\psi^{\mu \nu}, \psi^{\lambda}\right)^{T} \\
& =\left(\psi^{01}, \psi^{02}, \psi^{03}, \psi^{23}, \psi^{31}, \psi^{12}, \psi^{0}, \psi^{1}, \psi^{2}, \psi^{3}\right)^{T},
\end{aligned}
$$

where $\psi^{\lambda}$ are real and $\psi^{\mu \nu}$ are purely imaginary [19]. Because of antisymmetry of $\psi^{\mu \nu}$ we have $p_{\nu} \psi^{\nu}=0$ and this is spin 1 condition. The set of (7) was first written by Proca [26].

It turns out that in the case of the more general equation (2) there are also other representations of $\rho^{\mu}$ matrices; see [23, 24] for a review. For example, there are two representations 7 for which the corresponding $7 \times 7$ matrices $\rho^{\mu}$ yield the Hagen-Hurley equations for spin 1 bosons [16-18]; see also $[19,23,24]$. There are also two sets of $3 \times 3$ matrices $\rho^{\mu}$ obeying (2); see [27]. In the present work we find a simple relation between DKP and Tzou representations.

Several comments concerning equivalence/nonequivalence of formalisms used to describe vector bosons are in order here. The spin 1 DKP and Proca theories are equivalent in the free case as well as in the case of minimally coupled interactions. On the other hand, the DKP formalism permits nonstandard couplings via nonminimal interactions, which are not possible in the Proca theory; see $[28,29]$ and references therein. Finally, the Hagen-Hurley equations are yet another formalism since these equations violate parity and thus their applications are limited to weakly interacting particles [30].

\section{From Representation 5 of the DKP Algebra to Representations 3 of the Tzou Algebra}

As explained in the Introduction, we first carry out a unitary transformation $C$

$$
C=\frac{1}{\sqrt{2}}\left(\begin{array}{ccccc}
1 & 0 & 0 & 1 & 0 \\
0 & 1 & i & 0 & 0 \\
0 & 0 & 0 & 0 & \sqrt{2} \\
0 & -1 & i & 0 & 0 \\
1 & 0 & 0 & -1 & 0
\end{array}\right)
$$

which transforms the wave-function (6) into the spinor form:

$$
\Psi \longrightarrow C \Psi=\frac{1}{\sqrt{2}}\left(\psi^{0}+\psi^{3}, \psi^{1}+i \psi^{2}, \sqrt{2} \psi,-\psi^{1}+i \psi^{2}, \psi^{0}-\psi^{3}\right)^{T}
$$

i.e., involving spinor components $\psi^{A \dot{B}}$ rather than vector ones $\psi^{\mu}$; see [27] for the definition of $\psi^{A \dot{B}}$.

Then we apply two projection operators, $P=\operatorname{diag}(1,1$, $1,0,0)$ and $Q=\operatorname{diag}(0,0,1,1,1)$, projecting onto threedimensional subspaces, to define new matrices:

$$
\begin{aligned}
& \widehat{\rho}^{\mu}=\frac{1}{\sqrt{2}} P C \beta^{\mu} C^{-1} P, \\
& \check{\rho}^{\mu}=\frac{1}{\sqrt{2}} Q C \beta^{\mu} C^{-1} Q .
\end{aligned}
$$

The matrices $\widehat{\rho}^{\mu}$ which can be written in explicit compact form as

$$
\widehat{\rho}^{\mu} p_{\mu}=\left(\begin{array}{ccc|cc}
0 & 0 & p_{0}+p_{3} & 0 & 0 \\
0 & 0 & p_{1}+i p_{2} & 0 & 0 \\
p_{0}-p_{3} & -p_{1}+i p_{2} & 0 & 0 & 0 \\
\hline 0 & 0 & 0 & 0 & 0 \\
0 & 0 & 0 & 0 & 0
\end{array}\right),
$$

fulfilling the Tzou commutation relations (2). Matrices $\widehat{\rho}^{\mu}$ can be immediately read off from (12) as they are proportional 
to $p^{\mu}$ s. This result was obtained in [22] but for the whole operator $\hat{\rho}^{\mu} k_{\mu}$ only and not for individual matrices $\hat{\rho}^{\mu}$.

While these matrices belong to reducible representation of the Tzou algebra (2), they induce the irreducible representation 3:

$$
\rho^{\mu} p_{\mu}=\left(\begin{array}{ccc}
0 & 0 & p_{0}+p_{3} \\
0 & 0 & p_{1}+i p_{2} \\
p_{0}-p_{3} & -p_{1}+i p_{2} & 0
\end{array}\right) \text {. }
$$

The matrices $\check{\rho}^{\mu}$ lead to another irreducible representation 3 of the Tzou algebra. These two representations 3 were described in [27].

\section{From Representation 10 of the DKP Algebra to Representations 7 of the Tzou Algebra}

We start carrying out a unitary transformation $C$

C

$$
=\frac{1}{\sqrt{2}}\left(\begin{array}{cccccccccc}
1 & 0 & 0 & i & 0 & 0 & 0 & 0 & 0 & 0 \\
0 & 1 & 0 & 0 & i & 0 & 0 & 0 & 0 & 0 \\
0 & 0 & 1 & 0 & 0 & i & 0 & 0 & 0 & 0 \\
0 & 0 & 0 & 0 & 0 & 0 & \sqrt{2} & 0 & 0 & 0 \\
0 & 0 & 0 & 0 & 0 & 0 & 0 & \sqrt{2} & 0 & 0 \\
0 & 0 & 0 & 0 & 0 & 0 & 0 & 0 & \sqrt{2} & 0 \\
0 & 0 & 0 & 0 & 0 & 0 & 0 & 0 & 0 & \sqrt{2} \\
1 & 0 & 0 & -i & 0 & 0 & 0 & 0 & 0 & 0 \\
0 & 1 & 0 & 0 & -i & 0 & 0 & 0 & 0 & 0 \\
0 & 0 & 1 & 0 & 0 & -i & 0 & 0 & 0 & 0
\end{array}\right) \text {, }
$$

which transforms the wave-function (8), $\Psi \longrightarrow C \Psi$, into the form involving self-dual and antiself-dual tensors, $\psi_{S}^{\mu \nu}$ and $\psi_{A}^{\mu \nu}$. More exactly, $\psi_{S}^{\mu \nu}=\psi^{\mu \nu}+i \widehat{\psi}^{\mu \nu}$ and $\psi_{A}^{\mu \nu}=\psi^{\mu \nu}-i \widehat{\psi}^{\mu \nu}$, where a tensor $\widehat{\psi}^{\mu \nu}$ dual to a given antisymmetric tensor $\psi^{\mu \nu}$ is defined as $\widehat{\psi}_{\mu \nu} \stackrel{d f}{=}(i / 2) \epsilon_{\mu \nu \kappa \lambda} \psi^{\kappa \lambda}$ and $\widehat{\psi}_{S}^{\mu \nu}=\psi_{S}^{\mu \nu}, \widehat{\psi}_{A}^{\mu \nu}=-\psi_{A}^{\mu \nu}$. Self-dual and antiself-dual tensors $\psi_{S}^{\mu \nu}$ and $\psi_{A}^{\mu \nu}$ are directly related to symmetric spinors $\xi^{A B}$ and $\eta_{\dot{C} \dot{D}}[27,31,32]$.

Next, we apply projection operators, $P=\operatorname{diag}(1,1,1,1$, $1,1,1,0,0,0)$ and $Q=\operatorname{diag}(0,0,0,1,1,1,1,1,1,1)$, projecting onto seven-dimensional subspaces, to define new matrices:

$$
\begin{aligned}
& \hat{\rho}^{\mu}=\frac{1}{\sqrt{2}} P C \beta^{\mu} C^{-1} P, \\
& \check{\rho}^{\mu}=\frac{1}{\sqrt{2}} Q C \beta^{\mu} C^{-1} Q .
\end{aligned}
$$

The $10 \times 10$ matrices $\widehat{\rho}^{\mu}$ and $\check{\rho}^{\mu}$ are reducible representations of Tzou algebra (2) and induce irreducible representations 7.
For example, the matrices $\rho^{\mu}$ induced by $\hat{\rho}^{\mu}$ can be written in explicit compact form as

$$
\begin{aligned}
& \rho^{\mu} p_{\mu} \\
& \quad=\left(\begin{array}{ccccccc}
0 & 0 & 0 & p_{1} & p_{0} & i p_{3} & -i p_{2} \\
0 & 0 & 0 & p_{2} & -i p_{3} & p_{0} & i p_{1} \\
0 & 0 & 0 & p_{3} & i p_{2} & -i p_{1} & p_{0} \\
-p_{1} & -p_{2} & -p_{3} & 0 & 0 & 0 & 0 \\
p_{0} & -i p_{3} & i p_{2} & 0 & 0 & 0 & 0 \\
i p_{3} & p_{0} & -i p_{1} & 0 & 0 & 0 & 0 \\
-i p_{2} & i p_{1} & p_{0} & 0 & 0 & 0 & 0
\end{array}\right) .
\end{aligned}
$$

Equation $\rho^{\mu} p_{\mu} \Psi=m \Psi$ is unitarily equivalent to the Hagen-Hurley equation [19].

\section{Summary}

We have constructed transformations of the Duffin-KemmerPetiau matrices, corresponding to representations 5 and 10, inducing matrices of representations 3 and 7 of the Tzou algebra, respectively. These transformations consist of a (renormalized) constant unitary transformation and a projection operator onto lower-dimensional subspace; see (11a), (11b), (15a), and (15b). The computations have been carried out in a particular representation of the corresponding matrices. On the other hand, all the results are valid in all other representations which can be obtained via a unitary or similarity transformation of these matrices.

These Tzou representations arise in the process of splitting relativistic equations, leading to subsolutions of these equations. For example, representation 7 appearing in the Hagen-Hurley theory of spin 1 bosons [16-18] arises in the process of splitting the DKP equation written for representation 10 of $\beta^{\mu}$ matrices [19].

These simple formulae can be contrasted with complicated, momentum dependent, and similarity transformations described in [22]. Moreover, those similarity transformations transform correctly the whole DKP operator $\left(\beta^{\mu} k_{\mu}-m\right)$ only; see (12) in [22].

\section{Data Availability}

The manuscript is self-contained, no additional data have been necessary.

\section{Conflicts of Interest}

The author declares that there are no conflicts of interest regarding the publication of this paper.

\section{References}

[1] N. Kemmer, "The particle aspect of meson theory," Proceedings of the Royal Society A Mathematical, Physical and Engineering Sciences, vol. 173, no. 952, pp. 91-116, 1939. 
[2] R. J. Duffin, "On the characteristic matrices of covariant systems," Physical Review A: Atomic, Molecular and Optical Physics, vol. 54, no. 12, p. 1114, 1938.

[3] G. Petiau, University of Paris Thesis, published in: Acad. Royale de Belgique, Classe de Sci., Memoires Coll. in 8 o, 16 (1936) Fasc. 2 .

[4] F. Yaşuk, C. Berkdemir, A. Berkdemir, and C. Önem, "Exact solutions of the Duffin-Kemmer-Petiau equation for the deformed hulthen potential," Physica Scripta, vol. 71, no. 4, pp. 340-343, 2005.

[5] Y. Kasri and L. Chetouani, "Energy spectrum of the relativistic Duffin-Kemmer-Petiau equation," International Journal of Theoretical Physics, vol. 47, no. 9, pp. 2249-2258, 2008.

[6] Y. Chargui, A. Trabelsi, and L. Chetouani, "Bound-states of the $(1+1)$-dimensional DKP equation with a pseudoscalar linear plus Coulomb-like potential," Physics Letters A, vol. 374, no. 29, pp. 2907-2913, 2010.

[7] H. Hassanabadi, B. H. Yazarloo, S. Zarrinkamar, and A. A. Rajabi, "Duffin-Kemmer-Petiau equation under a scalar Coulomb interaction," Physical Review C, vol. 84, article 064003, 2011.

[8] M. Hamzavi and S. M. Ikhdair, "Any J-state solution of the Duffin-Kemmer-Petiau equation for a vector deformed woodssaxon potential," Few-Body Systems, vol. 53, no. 3-4, pp. 461-471, 2012.

[9] H. Hassanabadi, Z. Molaee, and S. Zarrinkamar, "DKP oscillator in the presence of magnetic field in $(1+2)$-dimensions for spin-zero and spin-one particles in noncommutative phase space," The European Physical Journal C, vol. 72, article 2217, 2012.

[10] A. Boumali and H. Hassanabadi, "The thermal properties of a two-dimensional Dirac oscillator under an external magnetic field," The European Physical Journal Plus, vol. 128, article 124, 2013.

[11] H. Hassanabadi, S. S. Hosseini, A. Boumali, and S. Zarrinkamar, "The statistical properties of Klein-Gordon oscillator in noncommutative space," Journal of Mathematical Physics, vol. 55, no. 3, article 100, Article ID 033502, 2014.

[12] M. Darroodi, H. Hassanabadi, and N. Salehi, "The modified Woods-Saxon potential in the Duffin-Kemmer-Petiau equation," The European Physical Journal A, vol. 51, no. 6, 2015.

[13] N. Salehi and H. Hassanabadi, "Scattering amplitude of the Duffin-Kemmer-Petiau equation for the Yukawa potential for J = 0," The European Physical Journal A, vol. 51, no. 8, 2015.

[14] L. P. de Oliveira, "Quantum dynamics of relativistic bosons through nonminimal vector square potentials," Annals of Physics, vol. 372, pp. 320-328, 2016.

[15] O. J. Oluwadare and K. J. Oyewumi, "Scattering state solutions of the Duffin-Kemmer-Petiau equation with the Varshni potential model," The European Physical Journal A, vol. 53, no. 2, 2017.

[16] C. R. Hagen and W. J. Hurley, "Magnetic moment of a particle with arbitrary spin," Physical Review Letters, vol. 24, no. 24, pp. 1381-1384, 1970.

[17] W. J. Hurley, "Relativistic wave equations for particles with arbitrary spin," Physical Review D: Particles, Fields, Gravitation and Cosmology, vol. 4, no. 12, pp. 3605-3616, 1971.

[18] W. J. Hurley, "Invariant bilinear forms and the discrete symmetries for relativistic arbitrary-spin fields," Physical Review D: Particles, Fields, Gravitation and Cosmology, vol. 10, no. 4, pp. 1185-1200, 1974.
[19] A. Okninski, "Non-standard solutions of relativistic wave equations and decays of elementary particles," https://arxiv.org/abs/ 1704.04236 .

[20] K. H. Tzou, Comptes rendus de l'Académie des Sciences (Paris), vol. 244, p. 2137, 1957.

[21] K. H. Tzou, "Corpuscular representation of the vector field, and comparison with the field of maximum spin 1 of the fusion theory," Journal de Physique et Le Radium, vol. 18, no. 11, pp. 619$624,1957$.

[22] A. Oknin'ski, "From Duffin-Kemmer-Petiau to Tzou algebras in relativistic wave equations," https://arxiv.org/abs/1801.04308.

[23] J. Beckers, N. Debergh, and A. G. Nikitin, "On parasupersymmetries and relativistic descriptions for spin one particles: I. The free context," Fortschritte der Physik, vol. 43, no. 1, pp. 67-80, 1995.

[24] J. Beckers, N. Debergh, and A. G. Nikitin, "On parasupersymmetries and relativistic descriptions for spin one particles. II. The interacting context with (electro)magnetic fields," Fortschritte der Physik, vol. 43, no. 1, pp. 81-96, 1995.

[25] A. Oknin'ski, "Effective Quark Equations," Acta Physica Polonica $B$, vol. 12, pp. 87-94, 1981.

[26] A. Proca, "Sur les equations fondamentales des particules elémentaires," Comptes Rendus Mathematique Academie des Sciences (Paris), vol. 202, pp. 1490-1492, 1936.

[27] A. Oknin'ski, "Splitting the Kemmer-Duffin-Petiau equations," Proceedings of Institute of Mathematics of NAS of Ukraine. https://arxiv.org/abs/math-ph/0309013.

[28] L. B. Castro and L. P. de Oliveira, "Remarks on the Spin-One Duffin-Kemmer-Petiau Equation in the Presence of Nonminimal Vector Interactions in (3+1) Dimensions," Advances in High Energy Physics, vol. 2014, Article ID 784072, 8 pages, 2014.

[29] H. Hassanabadi, Z. Molaee, M. Ghominejad, and S. Zarrinkamar, "Spin-one DKP equation in the presence of Coulomb and harmonic oscillator interactions in (1+3)-dimension," Advances in High Energy Physics, vol. 2012, Article ID 489641, 10 pages, 2012.

[30] A. Okniński, "Generalized Solutions of the Dirac Equation, W Bosons, and Beta Decay," Advances in High Energy Physics, vol. 2016, Article ID 2689742, 4 pages, 2016.

[31] J. Łopuszan'ski, Rachunek Spinorów (The Calculus of Spinors, in Polish), PWN-Polish Scientific Publishers, Warsaw, Poland, 1985.

[32] J. T. Łopuszański, “The representations of the Poincaré group in the framework of free quantum fields," Fortschritte der Physik, vol. 26, no. 4, pp. 261-288, 1978. 


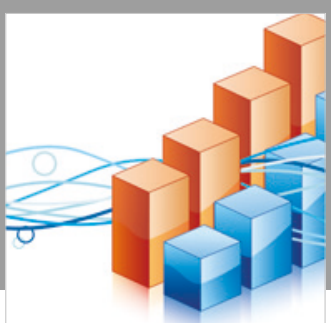

Advances in

Operations Research

\section{-n-m}
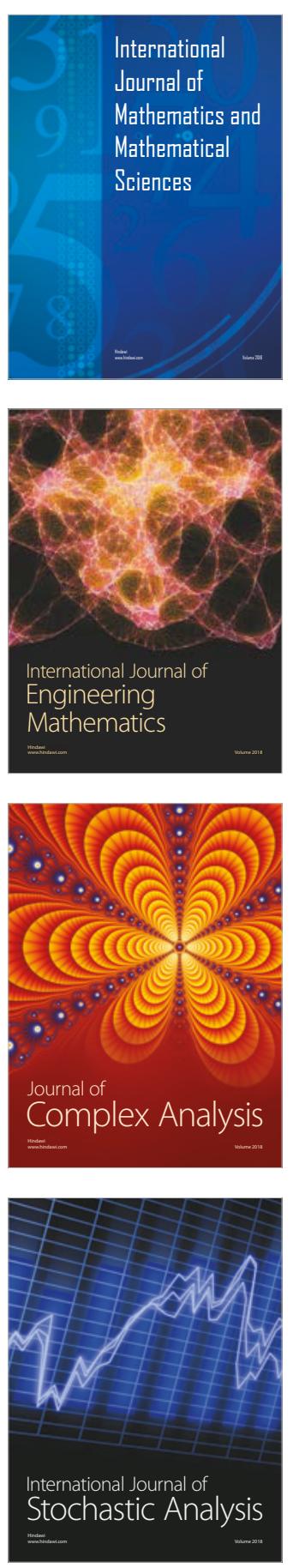
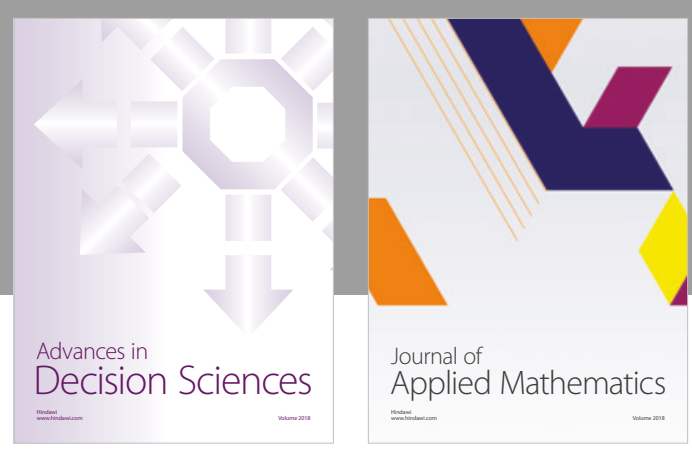

Journal of

Applied Mathematics
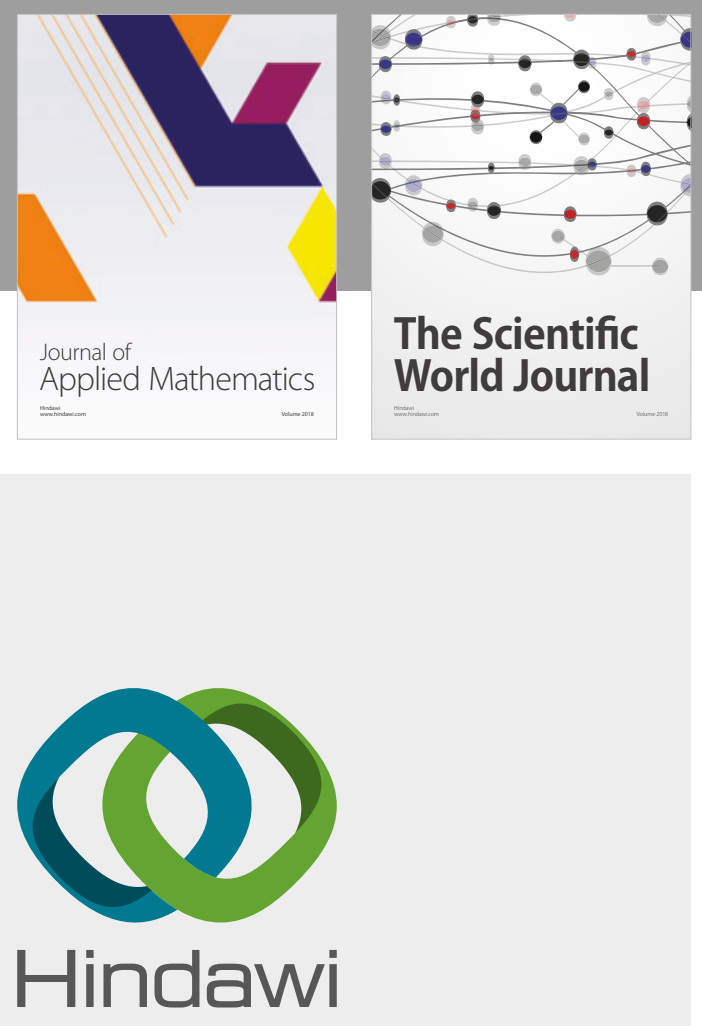

Submit your manuscripts at

www.hindawi.com

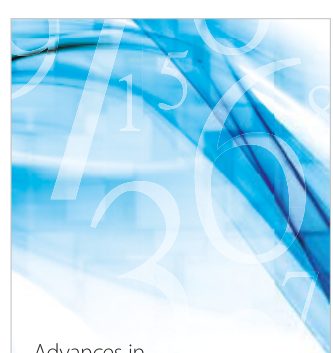

Advances in
Numerical Analysis
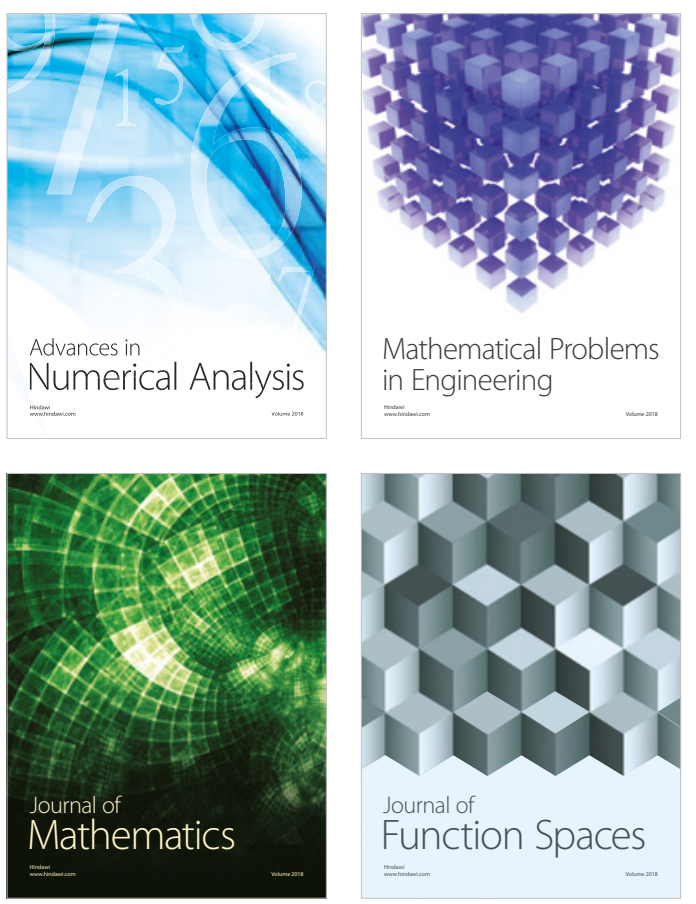

Mathematical Problems in Engineering

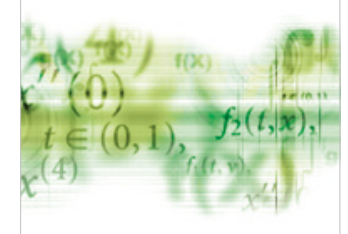

International Journal of

Differential Equations

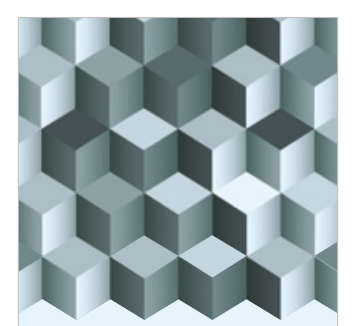

Journal of

Function Spaces

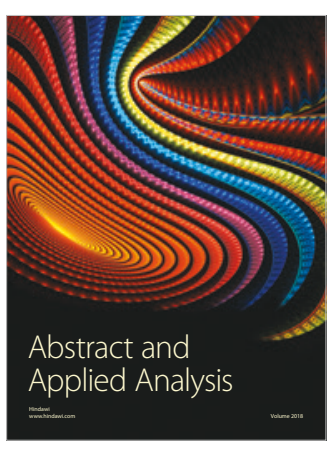

The Scientific

World Journal

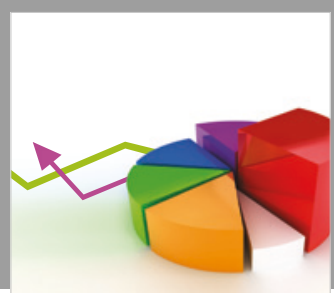

Journal of

Probability and Statistics
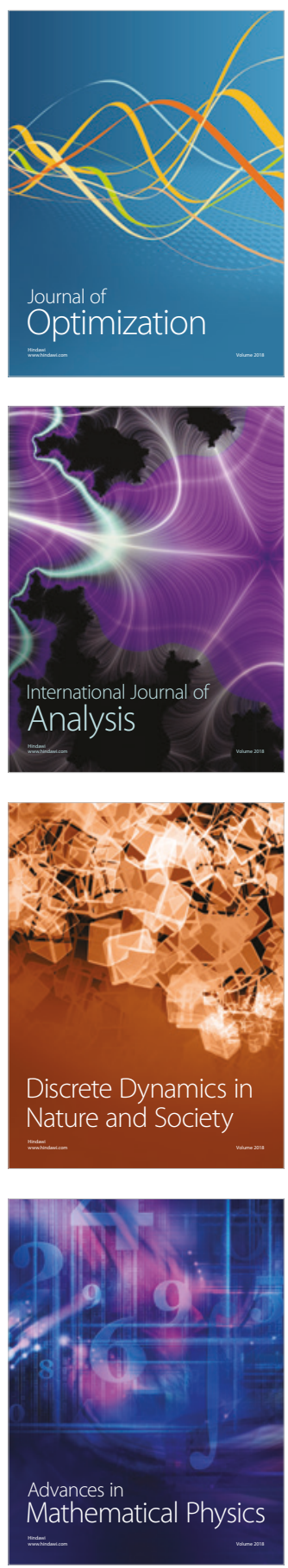